\title{
Arrangements of Oriented Hyperplanes
}

\author{
J. Linhart \\ Institut für Mathematik, Universität Salzburg, \\ Hellbrunnerstrasse 34, A-5020 Salzburg, Austria
}

\begin{abstract}
An arrangement of $n$ oriented hyperplanes or half-spaces divides $E^{d}$ into a certain number of convex cells. We study the number $c_{k}$ of cells which are covered by exactly $k$ half-spaces and derive an upper bound on $c_{k}$ for given $n$ and $d$.
\end{abstract}

\section{Introduction}

In the context of combinatorial or computational geometry, a finite set of hyperplanes in Euclidean or projective $d$-space is called an arrangement of hyperplanes. Such arrangements have been studied extensively, see, e.g., Chapter 18 in [8] and the major part of the book by Edelsbrunner [5]. Frequently, arrangements of oriented hyperplanes were also considered, but the orientation either played an auxiliary role (e.g., as in [10]) or was determined by the direction of the $d$ th coordinate axis [5] or the position of the origin [9].

Arrangements of oriented hyperplanes may be viewed as realizations of oriented matroids (see [2] and [7]). Presumably all the results of this paper can be generalized to arbitrary oriented matroids. However, the problems treated here seem to be more natural using "geometric language."

An arrangement of oriented hyperplanes in $E^{d}$ may be equivalently described by a finite set of (open) half-spaces $\left\{H_{1}^{+}, \ldots, H_{n}^{+}\right\}$. The complement of the union of the hyperplanes consists of a certain number of connected components which we call the cells of the arrangement [8]. If a cell is contained in exactly $k$ half-spaces $H_{i}^{+}$, we say it has weight $k$. It seems natural to ask for the number $c_{k}$ of cells with weight $k$, for each $k \in\{0, \ldots, n\}$. Let us call the $(n+1)$-tuple $\left(c_{0}, \ldots, c_{n}\right)$ the $c$-vector of the arrangement. So the central question may be formulated as follows.

Problem 1. Which $(n+1)$-tuples $\left(c_{0}, \ldots, c_{n}\right)$ of integers occur as $c$-vectors of arrangements of $n$ oriented hyperplanes in $E^{d}$ ? 
The relevance of this topic may be seen by comparing it with the closely related notion of levels in arrangements or the dual notion of semispaces of configurations of points [5], which seem to be of considerable importance in computational geometry, for example by their relation to Voronoi Diagrams of higher order (see, e.g., [9]). The essential difference between these notions and the present one is due to the special assumptions on the orientations (as indicated above), which lead to quite different results (see the remark after Conjecture 2 in Section 4).

Even in one dimension, Problem 1 is not at all trivial. Brehm [3] has found a complete characterization which is fairly complicated, but may be used to write a program which can decide rather quickly if a given $(n+1)$-tuple is the $c$-vector of an arrangement in $E^{1}$.

The general answer to Problem 1 seems to be extremely difficult. So we treat the following simpler question.

Problem 2. What is the maximal value of $c_{k}$ for given $n$ and $d$ ?

Let us denote this value by $C_{k}(n, d)$.

Observation 1. $C_{n-k}(n, d)=C_{k}(n, d)$ for $k \in\{0, \ldots, n\}$.

Proof. If $\left(c_{0}, \ldots, c_{n}\right)$ is the $c$-vector of a given arrangement, then $\left(c_{n}, \ldots, c_{0}\right)$ is the $c$-vector of the arrangement obtained by reversing the orientation of each hyperplane.

As a consequence of this observation we may restrict our investigations to $k \leq n / 2$.

Observation 2. $\quad C_{0}(n, d)=C_{n}(n, d)=1$.

$c_{n}=1$ characterizes the case where the interior of the intersection of all half-spaces $H_{i}^{+}$is not empty.

Observation 3. $\quad C_{k}(n, d)=\left(\begin{array}{l}n \\ k\end{array}\right)$ for $n \leq d$.

Proof. For $n \leq d$, let $H_{1}, \ldots, H_{n}$ be $n$ hyperplanes with linearly independent normal vectors. They may be transformed to a subset of the set of the coordinate hyperplanes of $E^{d}$ by a regular linear transformation. Thus each $n$-tuple $\left(\varepsilon_{1}, \ldots, \varepsilon_{n}\right) \in\{-1,1\}^{n}$ corresponds to a cell. The number of such $n$-tuples with exactly $k$ positive components is $\left(\begin{array}{l}n \\ k\end{array}\right)$.

The main result of this paper (Theorem 2) is a nontrivial upper bound on $C_{k}(n, d)$, which seems to be interesting especially for low dimensions. 
For the study of $C_{k}(n, d)$ we may restrict our attention to simple arrangements. An arrangement is called simple if no $d+1$ hyperplanes have a point or direction in common. Nonsimple arrangements may be transformed to simple ones by small displacements in such a way that none of the numbers $c_{k}$ decreases.

\section{One-Dimensional Euclidean Arrangements}

An oriented hyperplane in $E^{1}$ may be given by a pair $(x, \varepsilon)$ with $x \in \mathbb{R}$ and $\varepsilon \in\{+1,-1\}$. We associate the pair $(x,+1)$ with the interval $(x, \infty)$ and $(x,-1)$ with $(-\infty, x)$.

If $\mathscr{A}:=\left\{\left(x_{1}, \varepsilon_{1}\right), \ldots,\left(x_{n}, \varepsilon_{n}\right)\right\}$ is a simple arrangement of such hyperplanes, we assume $x_{1}<x_{2}<\cdots<x_{n}$. The combinatorial structure of $\mathscr{A}$, especially the $c$-vector, is then completely determined by the $n$-tuple $\left(\varepsilon_{1}, \ldots, \varepsilon_{n}\right)$. For convenience we set $x_{0}:=-\infty$ and $x_{n+1}:=+\infty$.

Proposition 1. $C_{k}(n, 1)=k+1$ for $0 \leq k \leq n / 2$.

Proof. Let $w_{i}$ be the weight of the cell $\left(x_{i}, x_{i+1}\right)$. Then

$$
w_{i}=\operatorname{card}\left\{j \mid j \leq i \text { and } \varepsilon_{j}=+1\right\}+\operatorname{card}\left\{j \mid j>i \text { and } \varepsilon_{j}=-1\right\} \text {. }
$$

Since $w_{i}=w_{i-1}+\varepsilon_{i}$ for each $i \in\{1, \ldots, n\}$,

$$
-1 \in\left\{\varepsilon_{i_{1}+1}, \ldots, \varepsilon_{i_{2}}\right\} \quad \text { if } \quad i_{1}<i_{2} \quad \text { and } \quad w_{i_{1}}=w_{i_{2}} .
$$

Now assume

$$
w_{i_{1}}=w_{i_{2}}=\cdots=w_{i_{m}}=k \quad \text { for } \quad 0 \leq i_{1}<i_{2}<\cdots<i_{m} \leq n .
$$

From (2) we conclude

$$
\operatorname{card}\left\{j \mid i_{1}<j \leq i_{m} \text { and } \varepsilon_{j}=-1\right\} \geq m-1,
$$

since between each two consecutive indices $i_{r}$ (including the greater one) there must be a $j$ with $\varepsilon_{j}=-1$. By (1) and (3),

$$
k=w_{i_{1}} \geq \operatorname{card}\left\{j \mid j>i_{1} \text { and } \varepsilon_{j}=-1\right\} \geq m-1 .
$$

This means $m \leq k+1$ and thus yields $c_{k} \leq k+1$. 
To establish equality, just consider the $n$-tuple $\left(\varepsilon_{1}, \ldots, \varepsilon_{n}\right)$ given by

$$
\varepsilon_{i}:=\left\{\begin{array}{lll}
+1 & \text { for } \quad i \leq 2 k \\
-1 & \text { for } \quad i \leq 2 k & \text { and } i \text { odd } \\
+1 & \text { for } i>2 k
\end{array}\right.
$$

Here $w_{0}=w_{2}=\cdots=w_{2 k}=k$.

\section{Spherical Arrangements}

To treat unbounded cells in two or more dimensions appropriately, we use spherical arrangements. An arrangement of oriented spherical hyperplanes in $S^{d}$ is nothing other than an arrangement of oriented hyperplanes in $E^{d+1}$ passing through the origin. However, in this case it is natural to consider opposite pairs of cells instead of single cells. Each cell $Z$ is characterized by its position vector $v(Z)=\left(v_{1}(Z), \ldots, v_{n}(Z)\right)[10],[5]$, which is defined by

$$
v_{i}(Z):= \begin{cases}+1 & \text { if } \quad Z \subset H_{i}^{+} \\ -1 & \text { if } \quad Z \subset H_{i}^{-}\end{cases}
$$

In a spherical arrangement, to each cell $Z$ there corresponds a cell $Z^{\prime}$ with $v\left(Z^{\prime}\right)=-v(Z)$, and then $\left\{Z, Z^{\prime}\right\}$ is called an opposite or complementary pair of cells. If $Z$ has weight $k$, then $Z^{\prime}$ has weight $n-k$. We denote the number of such pairs of cells by $s_{k}$ and the maximum of $s_{k}$ over all spherical arrangements of $n$ hyperplanes on $S^{d}$ by $S_{k}(n, d)$. Of course, Observations 1 and 2 hold for $S_{k}$ instead of $C_{k}$ also. Observation 3 has to be slightly modified:

Observation 3'. If $n \leq d+1$, then

$$
S_{k}(n, d)= \begin{cases}\left(\begin{array}{l}
n \\
k
\end{array}\right) & \text { for } k<n / 2 \\
\frac{1}{2}\left(\begin{array}{l}
n \\
k
\end{array}\right) & \text { for } k=n / 2 \quad \text { and } n \text { even }\end{cases}
$$

\subsection{One-Dimensional Spherical Arrangements}

We again begin with the one-dimensional case. Here a half-space is a semicircle. So an arrangement of oriented hyperplanes on $S^{1}$ may be viewed as a $2 n$-tuple $\left(\left(x_{1}, \varepsilon_{1}\right), \ldots,\left(x_{2 n}, \varepsilon_{2 n}\right)\right)$ with $x_{i} \in S^{1}, \varepsilon_{i} \in\{+1,-1\}$, such that the $x_{i}$ are in cyclic order and $x_{n+1}=-x_{i}, \varepsilon_{n+1}=-\varepsilon_{i}$ for $i \in\{1, \ldots, n\}$. If $\varepsilon_{i}=+1, x_{i}$ will be 
the starting point of the corresponding semicircle. For our purposes we may again omit the $x_{i}$ and concentrate on $\left(\varepsilon_{1}, \ldots, \varepsilon_{2 n}\right)$. The indices are understood modulo $2 n$.

\section{Proposition 2.}

$$
S_{k}(n, 1)= \begin{cases}k+1 & \text { for } k<(n-1) / 2 \\ 2 k+1=n & \text { for } k=(n-1) / 2 \text { and } n \text { odd }, \\ k & \text { for } k=n / 2 \text { and } n \text { even }\end{cases}
$$

Proof. The cells are circular arcs which we may denote like intervals: $z_{i}=$ $\left(x_{i}, x_{i+1}\right)$ for $i \in\{1, \ldots, 2 n\}$. The weight of $z_{i}$ is equal to the number of occurrences of -1 in the $n$-tuple $\left(\varepsilon_{i+1}, \ldots, \varepsilon_{i+n}\right)$. So if we set

$$
w_{i}:=\operatorname{card}\left\{j \in\{i+1, \ldots, i+n\} \mid \varepsilon_{j}=-1\right\},
$$

we have

$$
s_{k}=\operatorname{card}\left\{i \in\{1, \ldots, n\} \mid w_{i}=k \text { or } w_{i}=n-k\right\}
$$

Similarly to the Euclidean case, $w_{i}=w_{i-1}+\varepsilon_{i}$, and therefore $w_{i}=w_{j}$ with $i<j$ again implies $-1 \in\left\{\varepsilon_{i+1}, \ldots, \varepsilon_{j}\right\}$. This means that between each two values of $w$ which are both equal to $k$ or both equal to $n-k$, there must be at least one negative $\varepsilon$.

If $w_{i}=n-k$ and $w_{j}=k$ for some $i<j$, the tuple $\left(\varepsilon_{i+1}, \ldots, \varepsilon_{j}\right)$ must contain at least $n-2 k$ negative components. For $k<(n-1) / 2$ this means at least two.

Now let $w_{i_{1}}, \ldots, w_{i_{m}}$ be $m$ values of $w$ equal to $k$ or $n-k$, with

$$
0 \leq i_{1}<\cdots<i_{m}<n
$$

and

$$
t_{j}:=\operatorname{card}\left\{i \in\left\{i_{j}+1, \ldots, i_{j+1}\right\} \mid \varepsilon_{i}=-1\right\}
$$

Since all cells occur in complementary pairs, we may assume $w_{i_{1}}=k$. Then we get, for $k<(n-1) / 2$,

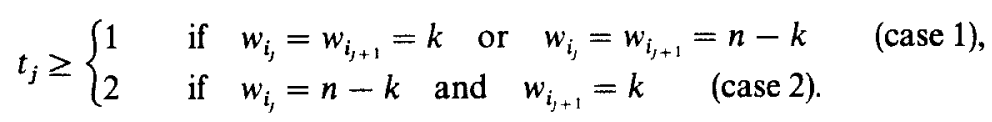

If $w_{i_{j}}=k$ and $w_{i_{j+1}}=n-k$, we can only say $t_{j} \geq 0$ (case 0 ). Because of the cyclic structure we might expect cases 0 and 2 to occur equally often, so that $t_{j} \geq 1$ in the mean. However, there is a slight problem for $j=m$. If we set $i_{m+1}=i_{1}+n$, 
we have $w_{i_{m+1}}=w_{i_{1}+n}=n-w_{i_{1}}$. This means there may be one occurrence of case 0 which is only counterbalanced by case 1 instead of case 2 . Thus we actually get

$$
k=w_{i_{1}}=\sum_{j=1}^{m} t_{j} \geq m-1,
$$

which yields $m \leq k+1$.

If $n$ is even and $k=n / 2$, we have $k=n-k$, and there is no distinction between cases 0,1 , and 2 . Here $t_{j}$ is always $\geq 1$, and we get $k=\sum t_{j} \geq m$.

If $n$ is odd and $k=(n-1) / 2$ the asserted inequality is trivial, since the total number of complementary pairs is equal to $n$.

Equality is attained by the following arrangements:

For $k<(n-1) / 2$ take the same $\varepsilon_{i}$ as at the end of the proof of Proposition 1 (for $i \in\{1, \ldots, n\}$ ). Then

$$
w_{0}=w_{2}=\cdots=w_{2 k}=k, \quad w_{1}=w_{3}=\cdots=w_{2 k-1}=k+1,
$$

and

$$
w_{2 k+i}=k+i<n-k \quad \text { for } \quad i \in\{1, \ldots, n-2 k-1\} \text {. }
$$

So $s_{k}=k+1$.

For $k=\lfloor n / 2\rfloor$, simply set $\varepsilon_{i}:=(-1)^{i}$ for $i \in\{1, \ldots, n\}$. For even $n$ this yields

$$
w_{i}= \begin{cases}k & \text { if } i \text { is even } \\ k-1 & \text { if } i \text { is odd }\end{cases}
$$

For odd $n, w_{i}=k$ for odd $i$ and $w_{i}=n-k=k+1$ for even $i$.

\subsection{Spherical Arrangements in Two or More Dimensions}

An arrangement of oriented hyperplanes or half-spaces in $E^{d}$ or $S^{d}$ is called a $d$-arrangement for short. If $\left\{H_{1}^{+}, \ldots, H_{n}^{+}\right\}$is a $d$-arrangement, then, for each $i_{0} \in\{1, \ldots, n\}$, the $n-1$ intersections $H_{i}^{+} \cap H_{i_{0}}$ with $i \neq i_{0}$ form a $(d-1)$-arrangement, the cells of which are $(d-1)$-faces or facets of certain cells of the original arrangement. The facets of a cell $Z$ with weight $k$ have weight $k-1$ or $k$ (depending on whether $Z$ is contained in the half-space corresponding to the facet or not).

Since in a simple spherical $d$-arrangement of at least $d+1$ hyperplanes each 
cell has at least $d+1$ facets, we obtain

Theorem 1. The maximal number $S_{k}(n, d)$ of complementary pairs of cells with weight $k$ or $n-k$ in a spherical d-arrangement of $n$ oriented hyperplanes, $n>d$, satisfies the following recursive inequality:

$$
S_{k}(n, d) \leq[n /(d+1)]\left(S_{k}(n-1, d-1)+S_{k-1}(n-1, d-1)\right)
$$

where $S_{0}(n, d)=1$ and $S_{k}(n, 1)$ is given by Proposition 2 .

For $d=2$, this gives the following bounds:

\section{Corollary 1.}

$$
S_{k}(n, 2) \leq \begin{cases}(2 k+1) n / 3 & \text { if } k<(n-2) / 2 \\ (3 k+1) n / 3 & \text { if } \quad k=(n-2) / 2 \quad \text { and } n \text { even } \\ (2 k) n / 3 & \text { if } \quad k=(n-1) / 2 \text { and } n \text { odd } \\ (4 k-2) n / 3 & \text { if } \quad k=n / 2 \text { and } n \text { even. }\end{cases}
$$

This bound may be compared with a result of Sharir [12] on a similar problem, namely, the number $S_{\leq k}(n)$ of vertices having weight $\leq k$ in an arrangement of closed curves in the plane, when each pair of curves intersect in at most two points: $S_{\leq k}(n) \leq 26 n k$ for $k \geq 1$.

By induction on the dimension we easily get the following somewhat crude estimate:

\section{Corollary 2.}

$$
S_{k}(n, d) \leq\left(\begin{array}{c}
n \\
d+1
\end{array}\right) \frac{2^{d}}{(n-d)(n-d+1)}(2 k+1)
$$

and thus $S_{k}(n, d)=O\left(k n^{d-1}\right)$ for $k \geq 1$ and constant $d$.

Remark. For $d \geq 3$ and $k=o(n)$ this is not the correct order of magnitude. Using the random sampling method of Clarkson and Shor [4], it is possible to show $S_{k}(n, d)=O\left(k^{\lceil d / 2\rceil} n^{\lfloor d / 2\rfloor}\right)$ for $k=o(n)$. To achieve this, we have to specialize the general setting of [4] in the following way. For the set $S$ of objects (in the terminology of [4]) we take the given set of half-spaces. The set $\mathscr{F}$ of ranges is the set of all points on $S^{d}$. The relation $\delta$ is defined such that $F \delta X$ means " $F$ is contained in the intersection of the set $X$ of hyperplanes" if $F \in \mathscr{F}$ and $X$ is a subset of $S$ with $d$ elements. Then the above order of magnitude of $S_{k}(n, d)$ may be derived from Theorem 3.1 of [4] in the same way as Corollary 3.3, which deals with semispaces of configurations of points. 


\section{Euclidean Arrangements in Two or More Dimensions}

In a Euclidean $d$-arrangement the unbounded cells play a special role since they may have only $d$ facets, whereas the bounded cells must have at least $d+1$.

Let us use the following notations:

$a_{k}$ is the total number of facets with weight $k$.

$b_{k}$ is the number of cells with weight $k$ which have at least $d+1$ facets.

$u_{k}$ is the number of unbounded cells with weight $k$ which have only $d$ facets.

So $c_{k}=b_{k}+u_{k}$.

Since the facets of a cell with weight $k$ have weight $k-1$ or $k$, we have

$$
(d+1) b_{k}+d u_{k} \leq a_{k}+a_{k-1} .
$$

This implies

$$
(d+1) c_{k} \leq a_{k}+a_{k-1}+u_{k}
$$

The unbounded cells are in one-to-one correspondence to the cells of the spherical $(d-1)$-arrangement obtained by translating the given hyperplanes such that they pass through the origin. However, of two opposite unbounded cells at most one can have only $d$ facets, provided the number of hyperplanes is greater than $d$ and the arrangement is simple. Therefore

$$
u_{k} \leq S_{k}(n, d-1)
$$

and we see:

Theorem 2. The maximal number $C_{k}(n, d)$ of cells with weight $k$ in a Euclidean $d$-arrangement of $n$ oriented hyperplanes, $n>d$, satisfies the following recursive inequality:

$$
\begin{aligned}
C_{k}(n, d) \leq & {[n /(d+1)]\left(C_{k}(n-1, d-1)+C_{k-1}(n-1, d-1)\right) } \\
& +[1 /(d+1)] S_{k}(n, d-1),
\end{aligned}
$$

where $C_{0}(n, d)=1, C_{k}(n, 1)$ is given by Proposition 1 , and $S_{k}(n, d-1)$ may be estimated by Theorem 1 .

Applying Propositions 1 and 2 we immediately get explicit bounds for $d=2$ :

\section{Corollary 3.}

$$
C_{k}(n, 2) \leq \begin{cases}(2 k+1) n / 3+(k+1) / 3 & \text { for } k<(n-1) / 2 \\ (2 k+1) n / 3+(2 k+1) / 3 & \text { for } k=(n-1) / 2 \text { and } n \text { odd } \\ (2 k+1) n / 3+k / 3 & \text { for } k=n / 2 \text { and } n \text { even }\end{cases}
$$


For $k=1$ and $n>3$ this means $C_{1}(n, 2) \leq n$. This can be strengthened considerably:

Observation 4. $C_{1}(n, d)=n$ for all $n$ and $d$.

Proof. To prove $C_{1}(n, d) \leq n$ we use induction on $n$. Let $\left\{H_{1}^{+}, \ldots, H_{n}^{+}\right\}$be an arrangement with $c_{1} \leq n$. If we add a half-space $H_{n+1}^{+}$, the new cells of weight 1 are either of the form

$Z \cap H_{n+1}^{+}$, with $Z$ being an old cell of weight 0 , or

$Z \cap H_{n+1}^{-}$, with $Z$ being an old cell of weight 1 .

Since there is at most one old cell of weight 0 , we get $c_{1} \leq n+1$ for the new arrangement. Equality is attained, e.g., by the complements of the half-spaces defining a convex polytope.

We can again get a closed-form estimate for higher dimensions by induction:

\section{Corollary 4.}

$$
C_{k}(n, d) \leq\left(\begin{array}{l}
n+1 \\
d+1
\end{array}\right) \frac{2^{d}}{(n-d+1)(n-d+2)}(k+1)
$$

and thus $C_{k}(n, d)=O\left(k n^{d-1}\right)$ for $k \geq 1$ and constant $d$.

Again, the method of [4] yields $C_{k}(n, d)=O\left(k^{\lceil d / 2} n^{\lfloor d / 2\rfloor}\right)$ for $k=o(n)$.

Lower bounds on $C_{k}(n, d)$ can be obtained by the construction of special arrangements. We mention, especially, the following fact, because in this case there is some hope that it constitutes the exact value.

Observation 5. $\quad C_{2}(n, 2) \geq\lfloor 3 n / 2\rfloor$ for $n \geq 5$.

Proof. This inequality is implied by the corresponding one concerning semispaces [6], where equality holds. It may be seen directly in the following way. Consider a regular $n$-gon and number the sides consecutively in cyclic order. Then translate the lines of the even-numbered sides outward until they pass the points of intersection of the neighboring odd-numbered lines. If the lines are oriented so that the center of the $n$-gon is always in the negative half-plane, we get a 2-arrangement with $c_{2}=\lfloor 3 n / 2\rfloor$.

Conjecture 1. $\quad C_{2}(n, 2)=\lfloor 3 n / 2\rfloor$ for $n \geq 5$. 


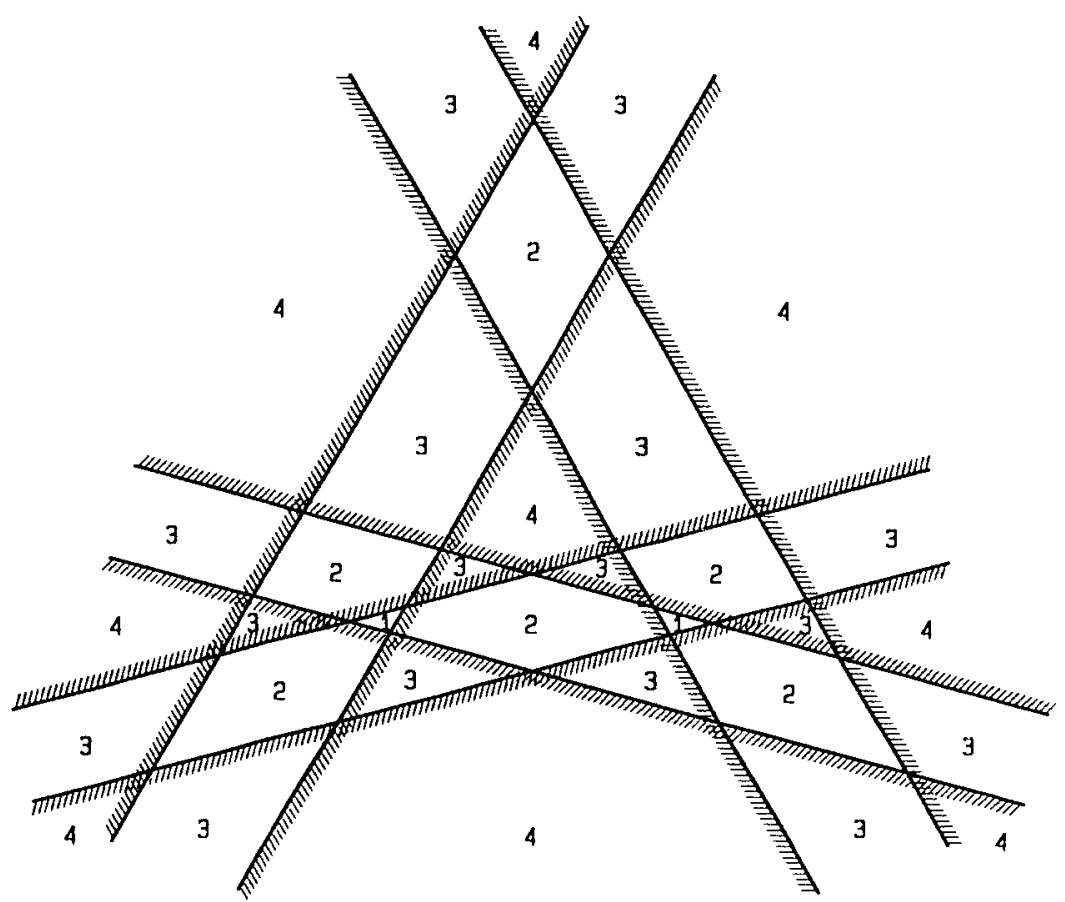

Fig. 1. An arrangement of eight oriented lines with 16 cells of weight 3 . Each cell is labeled by its weight.

Figure 1 shows a 2-arrangement of eight lines where the number of cells with weight 3 is possibly maximal. This would mean $C_{3}(8,2)=16$. It might be guessed that $C_{3}(n, 2) \leq 2 n$ for all $n$, with equality at least for $n=8$.

There is a simple example which shows that the bound of Corollary 3 has the correct order of magnitude at least for $k=n / 2$. Consider the half-planes $H_{i}^{+}$ given by the following inequalities (here $x_{1}, x_{2}$ denote the coordinates of a point in $E^{2}$ ):

$$
\begin{aligned}
& \text { For } i \in\{1, \ldots, n / 2\}: \\
& \text { if } i \text { is odd: } \quad x_{1}<i, \\
& \text { if } i \text { is even: } \quad x_{1}>i . \\
& \text { For } i \in\{n / 2+1, \ldots, n\}: \\
& \text { if } i \text { is odd: } \quad x_{2}<i, \\
& \text { if } i \text { is even: } \quad x_{2}>i .
\end{aligned}
$$

Here $c_{k}=(k+1)^{2} / 2>k n / 4$ for $n \equiv 2 \bmod 4$ and $k=n / 2$.

If we replace the sides of a regular $(n / 2)$-gon by pairs of oppositely oriented parallel lines at a suitable distance, we even get an example with

$$
c_{k}=(k+1)^{2}>k n / 2
$$


for $n \equiv 2 \bmod 4$ and $k=n / 2-1$. A picture of this arrangement for $n=10$ may be found in [11] (without orientations).

Such examples seem to support the following conjecture.

Conjecture 2. $C_{k}(n, 2)=\Theta(k n)$ for $0 \leq k \leq n / 2$ and all $n$.

This illustrates the difference between the concepts of semispaces (or $k$-sets) and arrangements of oriented hyperplanes. Let $M$ be a set of $n$ points in $E^{d}$. A $k$-set of $M$ is a subset of $M$ or cardinality $k$, which may be separated from its complement by a hyperplane. By duality, $k$-sets in the plane are equivalent to cells of weight $k$ in arrangements of nonvertical lines which are oriented from left to right [5]. It is known that the number of $k$-sets of $n$ points in the plane is $O(n \sqrt{k})[5],[6]$, in contrast to the above examples.

In spite of this difference there is an interesting theorem on semispaces in the plane [1] which may hold for arrangements of oriented lines as well:

Conjecture 3. Let $D_{k}(n, d):=\max \sum_{i=0}^{k} c_{i}$ over all c-vectors of arrangements of $n$ oriented hyperplanes in $E^{d}$. Then

$$
D_{k}(n, 2)=k n+1 \quad \text { for } \quad k<n / 2 \text {. }
$$

The proof of the corresponding theorem on semispaces uses the method of circular sequences of permutations (see [6]). It would be interesting to know whether this method can be adapted to general arrangements of oriented lines.

\section{Acknowledgment}

The author expresses his thanks to the referees, who drew his attention to papers [4] and [12].

\section{References}

1. N. Alon, E. Györi, The number of small semispaces of a finite set of points in the plane, J. Combin. Theory Ser. A 41 (1986), 154-157.

2. R. Bland, M. Las Vergnas, Orientability of matroids, J. Combin. Theory Ser, B 24 (1978), 94-123.

3. U. Brehm, A characterization of the $c$-vectors of arrangements of half-lines, Unpublished manuscript, Technical University, Berlin, 1991.

4. K. L. Clarkson, P. W. Shor, Applications of random sampling in computational geometry, II, Discrete Comput. Geom. 4 (1989), 387-421.

5. H. Edelsbrunner, Algorithms in Combinatorial Geometry, Springer-Verlag, Berlin, 1987.

6. H. Edelsbrunner, E. Welzl, On the number of line separations of a finite set in the plane, J. Combin. Theory Ser. A 38 (1985), 15-29.

7. J. Folkman, J. Lawrence, Oriented matroids, J. Combin. Theory Ser. B 25 (1978), 199-236.

8. B. Grünbaum, Convex Polytopes, Wiley, London, 1967. 
9. K. Mulmuley, On levels in arrangements and Voronoi diagrams, Discrete Comput. Geom. 6 (1991), 307-338.

10. G. Ringel, Teilungen der Ebene durch Geraden und topologische Geraden, Math. Z. 64 (1956), 79-102.

11. J. P. Roudneff, On the number of triangles in simple arrangements of pseudolines in the real projective plane, Discrete Math. 60 (1986), 243-251.

12. M. Sharir, On $k$-sets in arrangements of curves and surfaces. Discrete Comput. Geom. 6 (1991), 593-613.

Received August 15, 1991, and in revised form October 3, 1991, and December 7, 1992. 